\title{
Organic Thin Film Solar Cell Composed of Hetero-Deposited Langmuir-Blodgett Films
}

\author{
Atsushi AokI,* and Shinya Fukayama \\ Materials \& Science Engineering, Graduate School of Engineering, Nagoya Institute of Technology (Gokiso, Showa- \\ ku, Nagoya 466-8555, Japan)
}

Received December 18, 2009 ; Accepted December 20, 2009

\begin{abstract}
Organic thin film solar cells composed of the hetero-deposited Langmuir-Blodgett (LB) films were fabricated using poly(3-hexylthiophene) (P3HT) as a donor, [6,6]-phenyl C61 butyric acid methyl ester (PCBM) as an acceptor and poly(N-dodecylacrylamide) (pDDA) as an amphiphilic polymer. Various types of the solar cell such as the bulk heterojunction $(\mathrm{BHJ})$ type, $\mathrm{p}-\mathrm{n}$ heterojunction type, double layer $\mathrm{BHJ}$ type and P3HT single layer type solar cells were constructed and their photovoltaic properties were compared with them. The short circuit current densities observed at all the solar cells fabricated by LB technique were three orders of magnitude smaller than that of BHJ solar cell fabricated by spin-coating method because of the film resistance of the existence of pDDA for forming LB film. The open circuit voltage is interestingly obtained to be $1.23,0.82$ and $1.06 \mathrm{~V}$ at the P3HT single layer type, p-n heterojunction type and the double layer BHJ type solar cells fabricated by LB technique, resulting to be higher than $0.52 \mathrm{~V}$ at the BHJ type solar cell. The phenomena demonstrate that the hole produced by photoinduced charge separation can be accumulated at the P3HT layer of the solar cells.
\end{abstract}

Key Words : Organic Thin Film Solar Cell, Langmuir-Blodgett Film, poly(3-hexylthiophene), PCBM

\section{Introduction}

Organic thin film solar cells have been greatly investigated from the appearance of the bulk heterojunction (BHJ) solar cell using P3HT and PCBM. ${ }^{1)}$ The bicontinuous film structure has favorable structure for the photoinduced charge separation and the charge transport. However, the open circuit voltages (Voc) are reported to be lower than the thermodynamic voltage of $1.3 \mathrm{~V}$ calculated from the difference between HOMO of P3HT(-5.0 $\mathrm{eV})$ and LOMO of PCBM $(-3.7 \mathrm{eV}){ }^{2)}$ The lower Voc might be due to the film resistance, the recombination of hole and electron. The Voc is produced by accumulation of hole and electron produced by light irradiation at the ITO anode and $\mathrm{Al}$ cathode, respectively. In the $\mathrm{BHJ}$ solar cell, the bicontinuous structure is formed through the whole film. Thus, the recombination of hole in HOMO of P3HT and electron in LUMO of PCBM would take place at both of the anode and cathode, resulting decrease in Voc. One approach to overcome this problem is to prepare the multilayer device structure, where BHJ layer is sandwiched by hole-only and electron-only transporting layers. However, it is difficult to make this multilayer solar cell using wet process such as spin-coating method because of the solvent solubility of each layer. On the other hand, Langmuir-Blodgett (LB) technique enables to construct the multilayer device structure because solid phase monolayer on a water surface is transferred onto solid substrates. ${ }^{3)}$ The photovoltaic cells composed of single layer chlorophyll LB films ${ }^{4)}$ and fullerene derivative LB films ${ }^{5)}$ have been reported so far. In this study, various types of hetero-deposited LB structure were fabricated to improve Voc for the solar cells.
Their solar cell performances were investigated in order to achieve higher Voc near the theoretical voltage.

\section{Experimental}

pDDA was prepared by free radical polymerization of $N$-dodecylacrylamide monomer. P3HT and PCBM were purchased commercially. Surface pressure and molecular area isotherms were measured with USI LB trough at $20^{\circ} \mathrm{C}$. The appropriate amount of $1.0 \mathrm{mM}$ chloroform solution was dropped on a water surface and remained until evaporation of chloroform. The mixed P3HT monolayer at 0.67 mole fraction of P3HT in the mixture of P3HT and pDDA was transferred to ITO electrode at deposition pressure of $25 \mathrm{mN} / \mathrm{m}$. The mixed P3HT and PCBM monolayer at 0.46 and 0.04 mole fractions of P3HT and PCBM in the mixture of P3HT, PCBM and pDDA was transferred to the substrates at deposition pressure of $20 \mathrm{mN} / \mathrm{m}$. Glass substrate, silicon wafer and ITO electrode were used as the substrate. UV-vis spectroscopy was performed with HITACHI U2800 spectrometer. AFM images were observed by Molecular Imaging Picoscan 5. The hetero-deposited LB films on ITO electrodes were fabricated by LB deposition of mixed P3HT monolayer as a hole-transporting layer on ITO electrode and of mixed P3HT and PCBM monolayer as a photoinduced charge separation layer, successively. The solar cells were fabricated by vacuum vapor deposition of $\mathrm{LiF}$ as a hole blocking layer and $\mathrm{Al}$ as a cathode to the resulting hetero-deposited LB films through the shadow mask for restricting the active area of $0.20 \mathrm{~cm}^{2}$, successively. The film thickness of $\mathrm{LiF}$ and $\mathrm{Al}$ is 0.5 and 100 $\mathrm{nm}$, respectively. The solar cells were evaluated at room 
temperature in an air condition. I-V measurement was performed using a Keithly 2400 and $100 \mathrm{~mW} / \mathrm{cm}^{2}$ xenon lamp with AM 1.5G solar filter.

\section{Results and Discussion}

Figure 1 shows surface pressure and molecular area isotherms for single components and mixtures of P3HT, PCBM and pDDA at $20^{\circ} \mathrm{C}$. The monolayer behavior of the pristine $\mathrm{pDDA}$ shows that the surface pressure steeply raised with high collapse pressure of $45 \mathrm{mN} / \mathrm{m}$ and molecular occupied area was $0.3 \mathrm{~nm}^{2} /$ repeat unit, indicating formation of densely packed monolayer at the air/water interface. The monolayer behaviors of the pristine P3HT and PCBM show that the surface pressure raised a little even at the small molecular area, indicating monolayer is collapsed. Meanwhile, the monolayer behaviors of the mixtures of pDDA with P3HT or/ and PCBM show that the collapse pressures and the limiting surface area increase compared with those of the pristine P3HT and PCBM. These results suggest that the mixtures would form a monolayer on a water surface. The limiting area of P3HT and PCBM in the mixture monolayer is calculated to be $0.03 \mathrm{~nm}^{2} /$ repeat unit and $0.2 \mathrm{~nm}^{2} /$ molecule, respectively from area of the mixture and the limiting area of pDDA of $0.3 \mathrm{~nm}^{2} /$ repeating unit. The mixing weight ratio of P3HT and PCBM is selected to be $7 / 3$ from the literature where the best energy conversion efficiency is achieved in the BHJ solar cell prepared by spin-coating method. ${ }^{6}$ The limiting area of the monolayer mixed with three components is obtained to be $0.19 \mathrm{~nm}^{2}$, reasonably identifying with the calculated value of $0.17 \mathrm{~nm}^{2}$ from the additivity. In the following, the monolayers for the mixture of pDDA with P3HT or/and PCBM were transferred to the solid substrates to measure UV-vis spectra, AFM images and to prepare the solar cells.

Figure 2 shows UV-vis spectra for P3HT LB film, PCBM LB film and P3HT/PCBM LB film with 20 layers on glass substrates. The absorption is observed at P3HT LB film based on $\pi-\pi *$ transition of P3HT. The wavelength at the maximum absorption is $547 \mathrm{~nm}$ at the

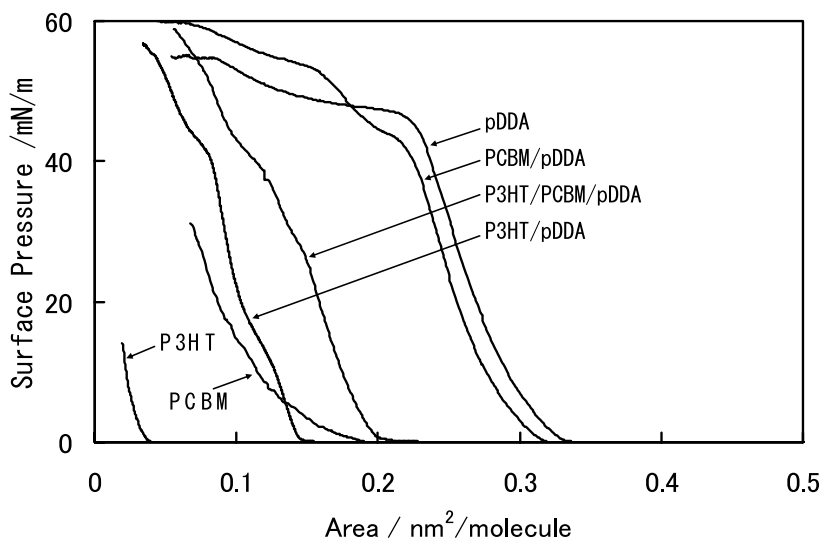

Fig. 1 Surface pressure-molecular area isotherms for single components and various mixtures of P3HT, PCBM and $\mathrm{pDDA}$ at $20^{\circ} \mathrm{C}$.
P3HT LB film, which is red-shifted compared with 524 $\mathrm{nm}$ of the spin-coated film, indicating longer $\pi$-conjugation length of P3HT. The absorption peak at $337 \mathrm{~nm}$ is observed at PCBM LB film, identical to PCBM in solution. There is a broad absorption shoulder in PCBM LB film. The absorption spectrum of the P3HT/PCBM LB film shows absorption peaks ascribing to P3HT and PCBM, indicating that P3HT and PCBM are mixing uniformly in the P3HT/PCBM LB film. The absorption shape of P3HT/PCBM LB film is similar to the combination of those of P3HT LB film and PCBM LB film. In fact, the absorbance at $550 \mathrm{~nm}$ of 0.14 at $\mathrm{P} 3 \mathrm{HT} / \mathrm{PCBM}$ LB film is reasonably in agreement with calculated value from that of 0.28 at P3HT LB film.

Figure 3 shows AFM images for monolayer LB films of the pristine pDDA (A) and the mixtures of pDDA with P3HT at 0.5 mole fraction of P3HT (B), with PCBM at 0.2 mole fraction of PCBM (C) and with P3HT and PCBM at 0.46 and 0.04 mole fraction of P3HT and PCBM (D), respectively. The AFM image of the pristine pDDA monolayer LB film on silicon wafer (A) shows molecularly smooth and flat surface within $0.5 \mathrm{~nm}$ roughness in the area of $2 \mu \mathrm{m} \times 2 \mu \mathrm{m}$. Meanwhile, the AFM images of three mixture monolayer LB films show phase separation and partially collapsed monolayer surface. There are dendritic structure and white circle spots in the image of the P3HT LB film (B). P3HT and pDDA phases would be separated in the monolayer because of immiscibility of polymers. The dendritic island and the flat sea indicate squeezed-out P3HT and pDDA monolayer, respectively. As the dendritic P3HT phase has $c a 2 \mathrm{~nm}$ height, it can regards as P3HT monolayer. The white circle spots have $c a 0.1 \mu \mathrm{m}$ diameter with $4-6 \mathrm{~nm}$ height, indicating aggregated P3HT. There is also dendritic structure in the AFM image for the PCBM LB film. The dendritic PCBM phase has ca $2 \mathrm{~nm}$ height. There is no white spot in the image for the PCBM LB film. It means that PCBM is squeezed out from pDDA monolayer but not produce aggregation. Unfortunately, the bicontinuous structure is not confirmed in the AFM image for P3HT/PCBM LB film because of less quantity of PCBM in the mixture. The image for P3HT/PCBM LB film is similar to that of P3HT LB film. The various types of

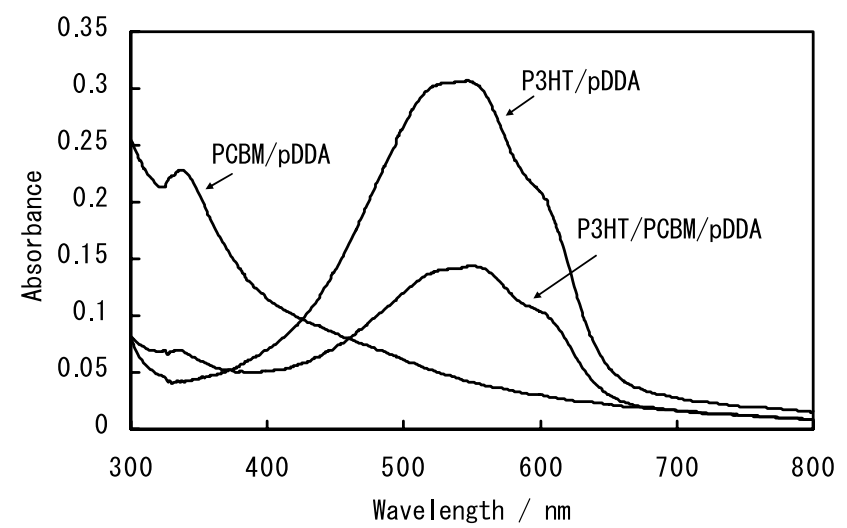

Fig. 2 UV-vis absorption spectra for various LB films with 20 layers on glass substrates. 


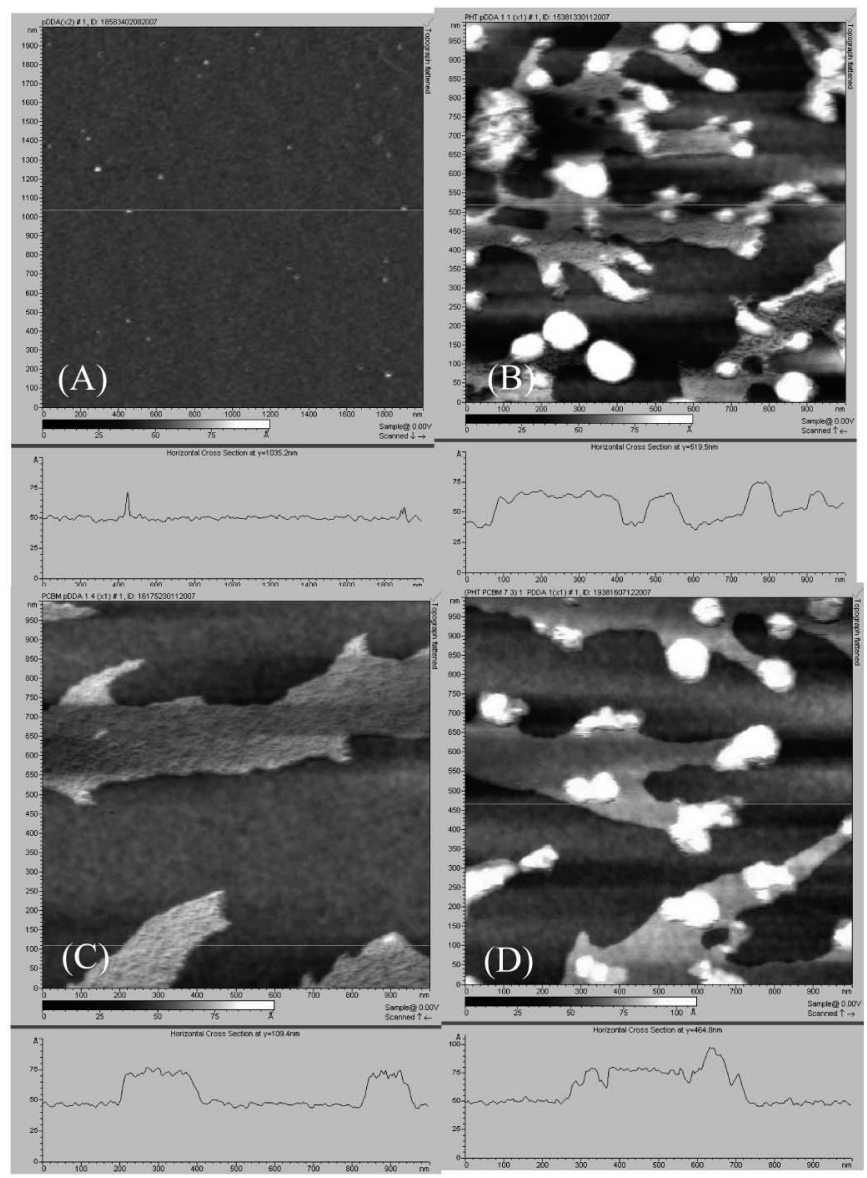

Fig. 3 AFM images for various monolayer LB films. $\mathrm{pDDA}(\mathrm{A}), \mathrm{P} 3 \mathrm{HT} / \mathrm{pDDA}(\mathrm{B}), \mathrm{PCBM} / \mathrm{pDDA}(\mathrm{C})$, and $\mathrm{P} 3 \mathrm{HT} /$ $\mathrm{PCBM} / \mathrm{pDDA}(\mathrm{D})$ on silicon wafers.

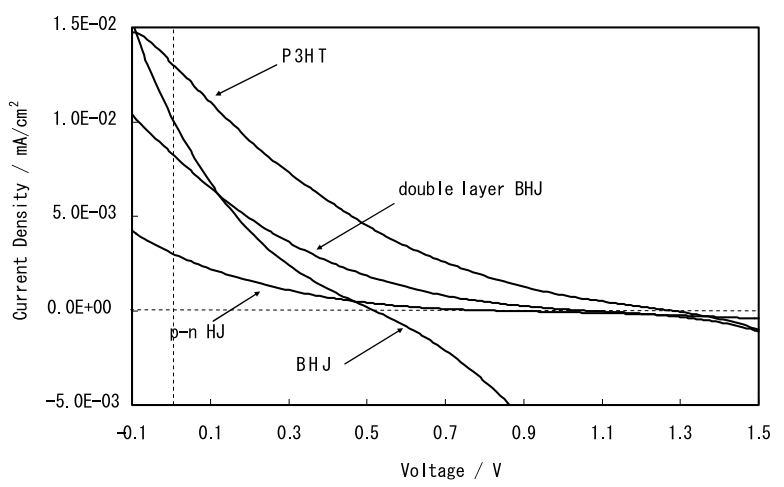

Fig. 4 I-V curves for various solar cells composed of LB films illuminated at $100 \mathrm{~mW} / \mathrm{cm}^{2}$ and AM $1.5 \mathrm{G}$.

solar cells using these LB films were fabricated and their performance was evaluated.

Figure 4 shows current density and voltage curves obtained at various types of the solar cells under simulated sun light irradiation. The number of the deposited monolayers was kept at constant value of 20 layers to compare with each type of the solar cells. BHJ solar cell was prepared by LB deposition of $\mathrm{P} 3 \mathrm{HT} / \mathrm{PCBM}$ monolayer until 20 layers. p-n HJ solar cell was fabricated by LB deposition of P3HT monolayer until 10 layers onto ITO substrate and by LB deposition of PCBM monolayer
Table 1 Various types of solar cell parameters

\begin{tabular}{lcccc}
\hline & $\mathrm{J}_{\mathrm{sc}}, \mu \mathrm{A} / \mathrm{cm}^{2}$ & $\mathrm{~V}_{\mathrm{oc}}, \mathrm{V}$ & $\mathrm{FF}$ & $\eta, \%$ \\
P3HT single layer & 9 & 1.23 & 0.15 & $1.6 \times 10^{-3}$ \\
BHJ & 10 & 0.52 & 0.16 & $8.5 \times 10^{-4}$ \\
p-n HJ & 3 & 0.82 & 0.13 & $2.9 \times 10^{-4}$ \\
double layer BHJ & 8 & 1.06 & 0.12 & $1.1 \times 10^{-3}$ \\
\hline
\end{tabular}

until 10 layers onto the resulting P3HT LB film, successively. Double layer BHJ solar cell was prepared by LB deposition of P3HT monolayer until 6 layers onto ITO substrate and by LB deposition of P3HT/PCBM monolayer until 20 layers onto the resulting P3HT LB film, successively. Solar cell parameters, such as Jsc, Voc, fill factor (FF) and energy conversion efficiency $(\eta)$ were summarized in Table 1. Jsc for all solar cells fabricated by LB technique was three orders of magnitude smaller than that of the BHJ solar cell prepared by spin-coating method. ${ }^{6}$ Since pDDA is insulator, the film resistance becomes higher in the solar cells fabricated by LB technique as mentioned before. ${ }^{4}$ Furthermore, P3HT and PCBM are diluted by pDDA to make LB films. Fill factor is similar values of 0.15 in all solar cells. These values are lower than that of spin-coating solar cell because fill factor usually depends on the film resistance. On the other hand, Voc at P3HT single layer, p-n HJ and double layer BHJ type solar cells are higher than $0.52 \mathrm{~V}$ at the BHJ type solar cell. The difference in the construction of the solar cells is the existence of P3HT layer without PCBM onto ITO surface. Therefore, the phenomena demonstrate that the hole produced by photoinduced charge separation can be accumulated at the P3HT layer of the solar cells. The hole accumulation at the ITO anode enhances Voc.

As LB technique can make hetero-deposited structure of monolayer onto substrates, various types of solar cells were fabricated by LB deposition using P3HT and PCBM. Since the solar cells fabricated at LB films have higher film resistance than that of spin-coated film, Jsc becomes three orders of magnitude smaller. Higher Voc was established by the hetero-deposited LB films.

\section{Acknowledgement}

The present work was partly supported by the joint usage/research program of the Hydrogen Isotope Research Center, University of Toyama (HRC2009-04).

\section{References}

1) W. L. Ma, C. Y. Yang, X. Gong, K. Lee, and A. J. Heeger, Adv. Funct. Mater., 15, 1617 (2005).

2) C. H. Woo, B. C. Thompson, B. J. Kim, M. F. Toney, and J. M. Frechet, J. Am. Chem. Soc., 130, 16324 (2008).

3) A. Aoki, Y. Abe, and T. Miyashita, Langmuir, 15, 1463 (1999).

4) A. Desormeaux, J. J. Max, and R. M. Leblanc, J. Phys. Chem., 97, 6670 (1993).

5) J. Jin, L. S. Li, Y. Li, Y. J. Zhang, X. Chen, D. Wang, S. Jiang, and T. J. Li, Langmuir, 15, 4565 (1999).

6) J. Nakamura, K. Murata, and K. Takahashi, App. Phys. Lett., 87, 132105 (2005). 